\title{
KIF21A mutation in two Chinese families with congenital fibrosis of the extraocular muscles type 1 and 3
}

\author{
JINGCHANG CHEN * , QINGQING YE* , DAMING DENG, JIANHUA YAN, \\ HOUBIAN LIN, TAO SHEN and YING LIN \\ State Key Laboratory of Ophthalmology, Zhongshan Ophthalmic Center, \\ Sun Yat-Sen University, Guangzhou, Guangdong 510060, P.R. China
}

Received June 30, 2015; Accepted July 6, 2016

DOI: $10.3892 / \mathrm{mmr} .2016 .5624$

\begin{abstract}
Congenital fibrosis of the extraocular muscles (CFEOM) is a hereditary ocular disease and can be classified into three subtypes. The aim of the present study was to determine the genetic basis and describe the clinical phenotype of CFEOM type 1 and 3. Two Chinese families with CFEOM type 1 and 3 were identified. The patients and their family members were subjected to comprehensive ophthalmic examinations, including best-corrected visual acuity, slit-lamp examination, fundus examination, assessment of palpebral fissure size, levator function, ocular motility, and cover and forced duction tests. Genomic DNA was extracted from the leukocytes of venous blood samples collected from the two families and from 200 unrelated control subjects from the same population. Coding exons of the KIF21A gene were amplified using polymerase chain reaction analysis and sequenced directly in the two probands. The detected mutations were further analyzed in all available family members and the unrelated control subjects. A heterozygous mutation, c. $2860 \mathrm{C}>\mathrm{T}$ (p.R954W), in KIF21A was identified in the two families, and this was cosegregated with the presence of the diseases in the two families, however, it was absent in the 200 normal control subjects. Among the three affected family members with CFEOM1, differences were observed with regard to the presence of aberrant eye movement. The results indicated that, in the patients with CFEOM1 and CFEOM3, the disease was caused by the same KIF21A gene mutation. The KIF21A gene
\end{abstract}

Correspondence to: Dr Ying Lin, State Key Laboratory of Ophthalmology, Zhongshan Ophthalmic Center, Sun Yat-Sen University, 54 Xian Lie Nan Road, Guangzhou, Guangdong 510060, P.R. China

E-mail: lylytulip@126.com

*Contributed equally

Abbreviations: CFEOM, congenital fibrosis of the extraocular muscles; PCR, polymerase chain reaction

Key words: congenital fibrosis of the extraocular muscles, gene, mutation, phenotype, heterogeneity may be a major disease-causing gene for Chinese patients with CFEOM3. Phenotypic heterogeneity was observed in the patients with CFEOM1.

\section{Introduction}

Congenital fibrosis of the extraocular muscles (CFEOM) is a hereditary ocular motility disorder characterized by non-progressive restrictive ophthalmoplegia and ptosis (1). CFEOM can be classified into three types, based on their phenotypes and inheritance patterns: CFEOM type 1 (CFEOM1; OMIM 135700), type 2 (CFEOM2; OMIM602078) and type 3 (CFEOM3; OMIM 600638, 607034). Of these, CFEOM1 and CFEOM3 can be inherited in an autosomal dominant manner, whereas CFEOM2 is inheritable in an autosomal recessive manner. Three disease-causing genes, Homo sapiens kinesin family member 21A $(K I F 21 A)$, paired-like homeobox $2 \mathrm{a}$ (ARIX or PHOX2A) and tubulin $\beta 3$ (TUBB3) have been identified to be associated with CFEOM (2-4).

Known as 'classic CFEOM', CFEOM1 is the most common form of CFEOM. It is a distinct, non-syndromic, congenital cranial dysinnervation disorder, and is characterized by congenital non-progressive bilateral external ophthalmoplegia, manifesting as restricted vertical and horizontal ocular motility and ptosis, leading to droopy eyelids and a chin-up position of the head (5). Heterozygous missense mutations in KIF21A have been identified to cause the classic CFEOM1 phenotype, and sporadic cases result from de novo mutations in $K I F 21 A$. In the 38-exon gene, only three exons have been reported to harbor mutations: Exon $21(2860 \mathrm{C}>\mathrm{T}, 2861 \mathrm{G}>\mathrm{A}$, $2861 \mathrm{G}>\mathrm{T})$, exon $8(1067 \mathrm{~T}>\mathrm{C})$ and exon $20(2830 \mathrm{G}>\mathrm{C}$, $2839 \mathrm{~A}>\mathrm{G}, 2840 \mathrm{~T}>\mathrm{C}, 2840 \mathrm{~T}>\mathrm{G})(6,7)$.

CFEOM3 represents a form of CFEOM, which does not meet the classic criteria for CFEOM1 or CFEOM2. It is less common than CFEOM1 and, compared with CFEOM1, individuals with CFEOM3 demonstrate variable expressivity of CFEOM, and at least one affected individual lacks or has only unilateral ophthalmoplegia or ptosis, or has one or both eyes fixed above the midline, or has the ability to raise one or both eyes above the midline $(8,9)$. CFEOM3 is often autosomal dominant with variable penetrance. The majority of families with CFEOM3 have been mapped to 16qter (10-12) and result from a heterozygous mutation in TUBB3 (3). However in $9 \%$ of 
families, CFEOM3 is reported to map to the CFEOM1 locus and harbor KIF21A mutations (9).

The present study identified two Chinese families, one with CFEOM1 and one with CFEOM3. The genetic basis was examined, and the clinical phenotypes of CFEOM1 and CFEOM3 were described.

\section{Materials and methods}

Family recruitment and clinical evaluation. Two families with CFEOM were identified. Following the provision of informed consent from all participating individuals or their parents, each participating individual was subjected to comprehensive ophthalmic examinations. Venous blood samples were collected from eight members of the two families and from 200 unrelated control subjects from the same population. Genomic DNA was extracted from peripheral blood leucocytes using standard protocols. The present study was performed, according to the principles of the Declaration of Helsinki and approved by the Ethics Committee of Zhongshan Ophthalmic Center, Sun Yat-Sen University (Guangzhou, China).

The two families were referred to Zhongshan Ophthalmic Center, Sun Yat-Sen University, China in April 2015 for severely restrictive strabismus and ptosis, present in the probands. All eight family members, comprising IV:2, IV:3, IV:4, V:2 and V:3 in family 1 , and I:1, I:2 and II:1 in family 2 (Fig. 1), which included the four affected individuals and their asymptomatic parents or siblings, were subjected to comprehensive ophthalmic examinations (Table I), including routine ophthalmic examination and strabismus assessments. The primary position of gaze, ductions and versions with a cover test were analyzed and quantified in six diagnostic positions. The affected individuals underwent forced duction assessment under topical anesthesia. Globe retraction or aberrant movements were observed. The width of the palpebral fissure was measured. Levator function was measured from the upper lid margin during attempted supraduction from the infraducted position without recruitment of the frontalis muscle. Ptosis was defined by a $\geq 2 \mathrm{~mm}$ covering of the iris by the upper lid, and was graded as mild if the upper lid covered the iris above the upper pupillary margin, moderate if the lid occluded half the pupil and severe if the lid occluded more than half the pupil (13). Visual acuity and cycloplegic refraction were also determined.

Mutation screening and sequence analysis. A $5 \mathrm{ml}$ venous blood sample was collected from each participant for genomic DNA extraction. DNA extraction from peripheral blood leukocytes was performed using a DNA extraction kit (Qiagen, Inc., Valencia, CA, USA) with standard protocols. Ethylene diaminetetraacetic acid-treated tubes were used for blood collection. Exons and flanking exon-intron boundaries of the KIF21A gene (38 exons) were amplified using polymerase chain reaction (PCR) analysis with the primers (14) (Beijing Genomics Institute, Guangzhou, China). Briefly, PCR was performed in a $50 \mu \mathrm{l}$ reaction volume with $2 \mu \mathrm{l}$ each primer, $2 \mu \mathrm{l}$ DNA, $25 \mu \mathrm{l}$ buffer mix and $19 \mu 1 \mathrm{H}_{2} \mathrm{O}$. All reagents used for PCR were purchased from Takara Bio, Inc. (Tokyo, Japan). The cycling profile was as follows: One cycle at $94^{\circ} \mathrm{C}$ for $5 \mathrm{~min}$, followed by 40 cycles at $94^{\circ} \mathrm{C}$ for $45 \mathrm{sec}, 59^{\circ} \mathrm{C}$ for $45 \mathrm{sec}$ and $72^{\circ} \mathrm{C}$ for $45 \mathrm{sec}$, with a final cycle at $72^{\circ} \mathrm{C}$ for $10 \mathrm{~min}$ (14). The PCR products were sequenced from both directions using an ABI3730 automated sequencer (PE Biosystems, Foster City, CA, USA). The sequencing results were analyzed using Seqman (version 2.3; Technelysium Pty, Ltd., Brisbane, QLD, Australia). Variations were identified by aligning sequences to the reference sequences from the National Center for Biotechnology Information database (NCBI; http://www.ncbi.nlm.nih.gov/). Detected variations were further analyzed by cosegregation analysis in the eight available family members and the normal control subjects.

\section{Results}

Clinical findings. Family and personal histories were carefully reviewed. The two families were from the Guangdong province of China. The diagnosis of CFEOM1 was based on inheritance patterns and clinical phenotypes, and this family was designated as family 1 . In family 1 , there were six affected family members with CFEOM in five generations (Fig. 1). The three affected members available for investigation (IV:3, V:2, V:3) shared the typical clinical features of CFEOM1, which have been reported previously (5). These features included bilateral congenital non-progressive ptosis, severely impaired vertical motility with an inability to raise either eye above the horizontal midline and variable impaired horizontal motility, infraducted position in primary gaze, a chin-up position of the head and an absence of Bell's phenomenon. Synergistic convergence on attempted upgaze was also observed in IV:3 and V:2. Of the three patients, one exhibited nystagmoid movements in all directions of gaze, one exhibited nystagmoid movements only in upgaze and the third exhibited no nystagmoid movements. The forced duction test demonstrated marked restrictions in passive elevation of the globes in IV:3. Family members V:2 and V:3 were unable to participate in the forced duction test. IV:3 and $\mathrm{V}: 2$ had poor visual acuity, and V:3 was unable to be assessed for visual acuity. No notable findings were detected in the examinations of the anterior segments or fundus.

In family 2, there were no other affected family members in the four generations. The proband presented with the typical clinical features of CFEOM3. Specifically, the proband had unilateral, congenital, non-progressive ptosis, variable restricted upgaze and horizontal eye movements, and was able to elevate left eye above the midline. The chin-elevated position of the head and the absence of Bell's phenomenon were similar to the observations with CFEOM1. The clinical presentations of the four affected members are listed in Table I. Examples of the compensatory head position and primary images of the unaltered eyelid and nine-gaze positions of the probands are shown in Figs. 2 and 3. The range of extraocular movements and the position of the eyes in the primary position of the four affected members are shown in Fig. 4.

Mutation screening. Genetic analysis documented the presence of a heterozygous mutation, c. $2860 \mathrm{C}>\mathrm{T}$, in the $K I F 21 \mathrm{~A}$ gene within all four patients of the two families. This mutation was not observed in the unaffected family members or in the 200 unrelated control subjects from the same population (Fig. 5). 
Table I. Clinical presentations of the four affected members.

\begin{tabular}{|c|c|c|c|c|}
\hline Parameter & Family 1: IV:3 & Family $1: \mathrm{V}: 2$ & Family 1: V:3 & Family 2: II:1 \\
\hline Age (years) & 34 & 6 & 3 & 4 \\
\hline Gender & Male & Female & Male & Female \\
\hline Birth history & Full-term & Full-term & Full-term & Full-term \\
\hline \multicolumn{5}{|l|}{ BCVA (decimals) } \\
\hline OD & 0.4 & 0.2 & Unable & 0.1 \\
\hline $\mathrm{OS}$ & 0.3 & 0.2 & Unable & 0.2 \\
\hline \multicolumn{5}{|l|}{ Refractive error } \\
\hline OD & $+0.50 /+2.00$ & $+2.00 /+5.00$ & $+2.00 /+1.00$ & $-0.25 /+1.00$ \\
\hline OS & $+1.00 /+2.50$ & $+1.50 /+2.50$ & $+2.00 /+1.50$ & $+1.25 /+1.75$ \\
\hline Stereopsis (butterfly) & $\mathrm{Nil}^{\mathrm{a}}$ & Nil & Nil & Nil \\
\hline Strabimus & Hypo with Eso & Hypo with Eso & Нypo & Hypo with Eso \\
\hline \multicolumn{5}{|l|}{ Eye movement } \\
\hline OD & Restrictive UAHM & Fixed & $\begin{array}{c}\text { Restrictive } \\
\text { UAHM }\end{array}$ & $\begin{array}{c}\text { Restrictive UAHM } \\
\text { and HM }\end{array}$ \\
\hline OS & $\begin{array}{c}\text { Restrictive UAHM } \\
\text { and HM }\end{array}$ & Fixed & $\begin{array}{l}\text { Restrictive } \\
\text { UAHM }\end{array}$ & $\begin{array}{c}\text { Variable restrictive } \\
\text { upgaze and HM }\end{array}$ \\
\hline \multicolumn{5}{|l|}{ Ptosis } \\
\hline OD & Moderate & Severe & Severe & No \\
\hline OS & Severe & Severe & Severe & Severe \\
\hline Bell's phenomenon & Absent & Absent & Absent & Absent \\
\hline \multicolumn{5}{|l|}{ Forced duction test } \\
\hline OD & Positive & Unable & Unable & Unable \\
\hline $\mathrm{OS}$ & Positive & Unable & Unable & Unable \\
\hline Nystagmus & On upgaze & In all directions of gaze & Absent & Absent \\
\hline Synergistic convergence & On upgaze & In all directions of gaze & Absent & Absent \\
\hline Pupil size & Normal & Normal & Normal & Normal \\
\hline Pupillary light reflex & Normal & Normal & Normal & Normal \\
\hline Compensatory head position & Chin up & $\begin{array}{c}\text { Chin up and mouth } \\
\text { open }\end{array}$ & Chin up & Chin up \\
\hline Proptosis ocular & No & No & No & No \\
\hline Upper eyelid lag & No & No & No & No \\
\hline Other ophthalmic examinations & Unremarkable & Unremarkable & Unremarkable & Unremarkable \\
\hline
\end{tabular}

${ }^{a}$ The result of 'nil' in the stereopsis test indicates that the patient cannot identify any figures present on the test, and the patient has no stereopsis. BCVA, best corrected visual acuity; OD, oculus dexter (the right eye); OS, oculus sinister (the left eye); Hypo, hypotropia; Eso, esotropia; UAHM, upgaze above horizontal midline; HM, horizontal motility.

\section{Discussion}

In the present study, a known mutation (c.2860C $>\mathrm{T}$ ) in $K I F 21 \mathrm{~A}$ was identified in two Chinese families with CFEOM1 and CFEOM3. The clinical findings revealed the phenotypic heterogeneity present within the three members of family 1 affected with CFEOM1.

The diagnosis of CFEOM1 in family 1 was based upon an autosomal dominant inheritance pattern, and was characterized by bilateral congenital ptosis, bilateral infraducted globe position in the primary gaze and a severely restricted upgaze with variable horizontal eye movements. In CEEOM1, all affected members of a family exhibit classic presentations of CFEOM and show dominant inheritance. By contrast, in families with
CFEOM3, one or more affected individuals fail to exhibit the classic characteristics of this disorder. For example, the individual's eyes may not be infraducted or may elevate above the midline, the individual may be unilaterally affected, or ptosis may be absent. In the proband of family 2 , unilateral ptosis and variable restrictive upgaze indicated a diagnosis of CFEOM3, rather than CFEOM1. Among the three affected members in family 1, IV:3 and V:2 exhibited nystagmus and synergistic convergence, whereas no such aberrant eye movements were recorded in V:3. It appears likely that aberrant innervation may be responsible for the severity of restricted eye movements. Among the three affected members in family 1, both IV:3 and $\mathrm{V}: 2$ exhibited nystagmus as well as synergistic convergence, whereas no such aberrant eye movements were present in $\mathrm{V}: 3$. 


\section{Family 1:}

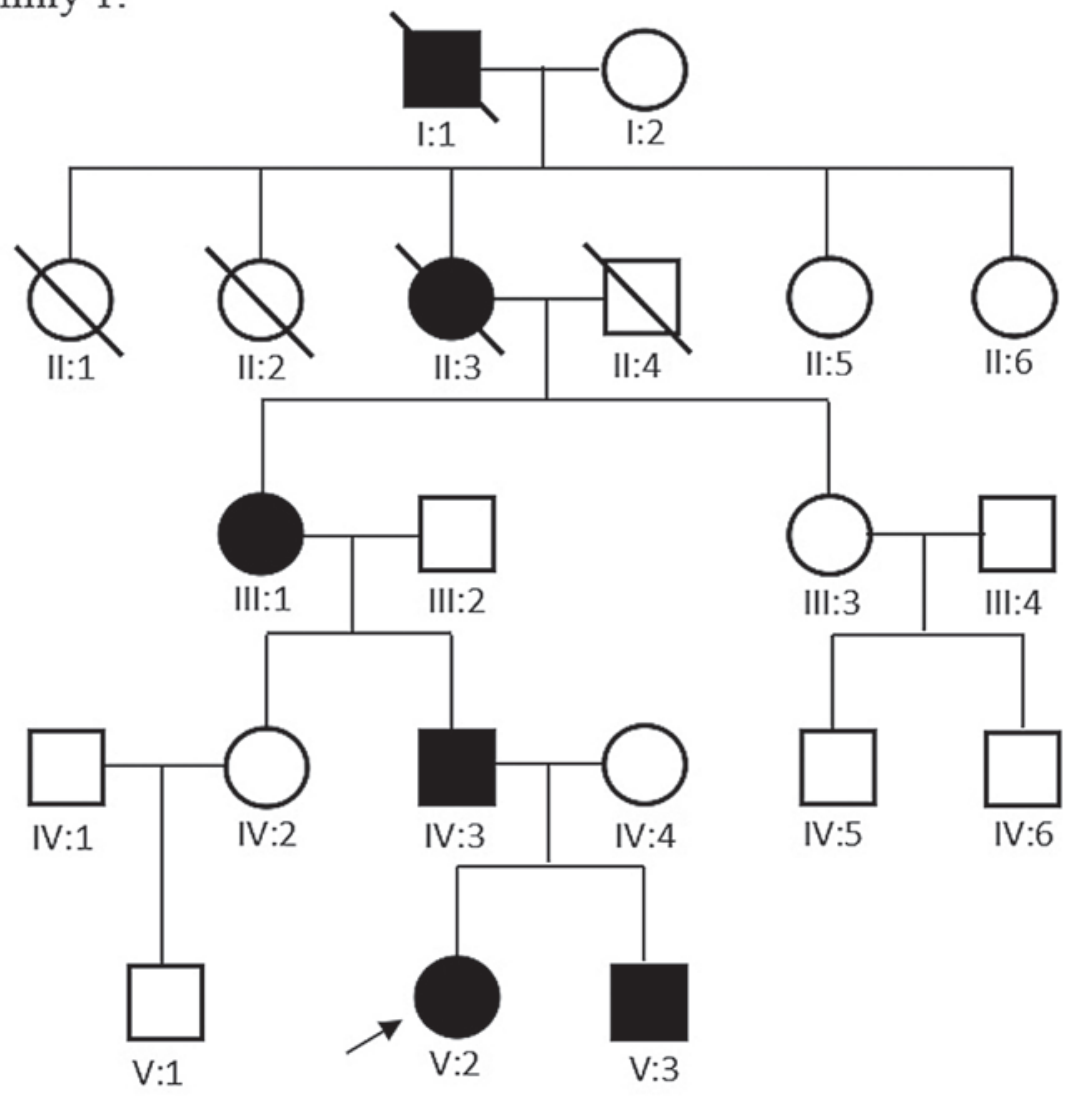

\section{Family 2:}

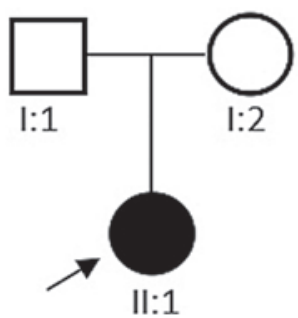

Figure 1. Pedigrees of the two families identified with congenital fibrosis of the extraocular muscles. Squares represent males, circles represent females, arrows indicate probands, and black symbols identify clinically affected individuals.

CFEOM1 is inherited as a fully penetrant, autosomal dominant trait and maps to the CFEOM1 locus on chromosome 12cen (15-18). The disease-causing gene, KIF21A, belongs to a family of kinesin motor proteins, which are responsible for the transport of membranous organelles, protein complexes and mRNAs to specific destinations within the cell, in a microtubule and ATP-dependent manner. These functions are essential for the normal morphogenesis and functioning of the cell (19). Results from previous studies have indicated that the KIF $21 \mathrm{~A}$ gene product may have a generalized effect in the development of all the ocular motor nuclei of cranial nerves III, IV and VI $(6,7)$.

To date, 12 missense mutations and one deletion have been identified in the KIF21A gene in patients presenting with CFEOM1 $(4,5,7,20,21)$. Among these, the c.2860C $>$ T mutation has been identified as the most common mutation, with a prevalence of between 72 and $75 \%(4,22)$. The c.2860C $>$ T mutation is located in a methylated $\mathrm{CpG}(\mathrm{mCpG})$ dinucleotide of the gene (23) and these $\mathrm{mCpG}$ sequences represent mutational hotspots in human genetic diseases. Random deamination of 5-methylcytosines produces these mutations (24). Considerable evidence exists indicating that the CFEOM1 phenotype results from mutations in $K I F 21 A$ and that sporadic cases are due to de novo mutations in the same gene $(7,19)$. The occurrence of this mutation in the families in the present study suggests a mutation hot spot.

The proband in family 2 also harbored the most commonly observed KIF21A mutation, c. $2860 C>$ T. KIF21A mutations are reported to be a rare cause of CFEOM3 (9). However, the $K I F 21 A$ mutation has been reported in a Taiwanese pedigree (25) and in families from other regions of China with CFEOM3 (5). To date, five Chinese families with CFEOM3 have been reported with identified mutations (5,25-27), with four sharing the same KIF21A mutation and the fifth harboring the TUBB3 gene mutation. This suggests that the KIF21A mutation may be a hot spot mutation of CFEOM3 in Chinese individuals. 
A

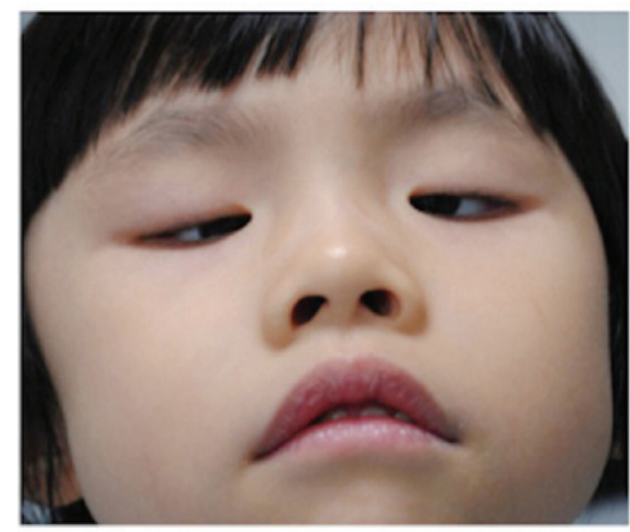

C

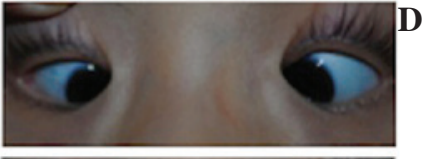

F

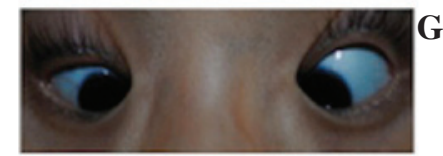

I

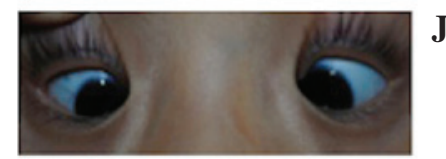

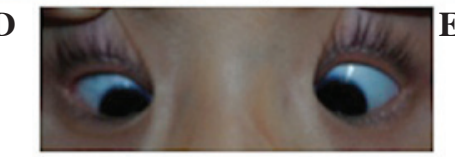
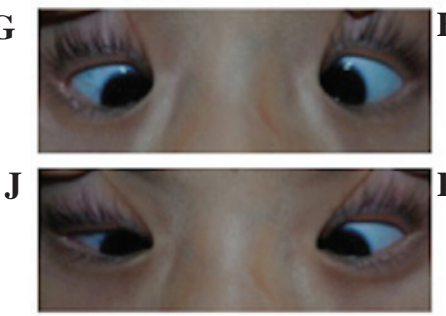

B
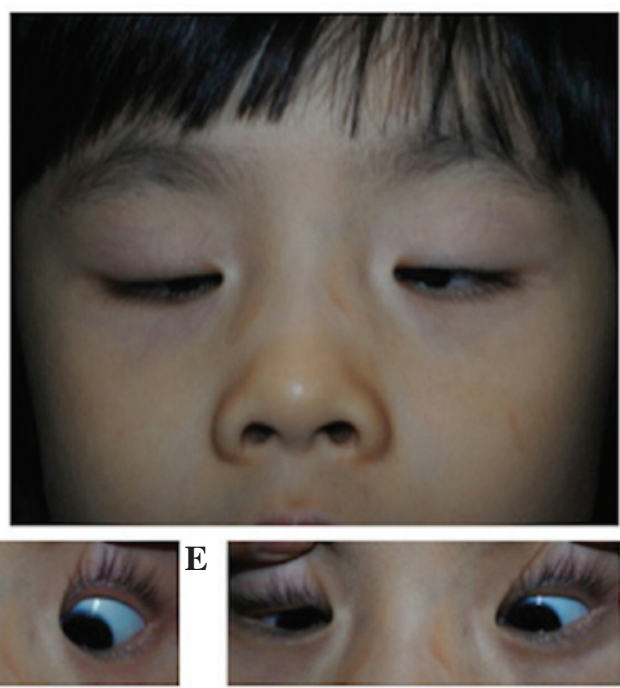

H
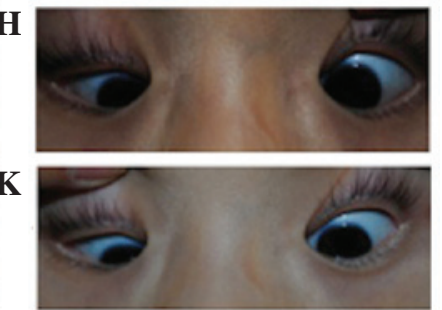

Figure 2. Images of the proband from family 1. Images were captured of the (A) compensatory head position, in (B) primary gaze with unaltered eyelids and in (C-K) nine-gaze positions with lifted eyelids (in sequence C-K, looking up and right, straight up, up and left, straight right, straight, straight left, down and left, straight down, down and left). This individual exhibited typical CFEOM1 features, including bilateral ptosis, infraducted globe position in primary gaze, severely restricted upgaze with an inability to raise the eyes above horizontal midline, variable impaired horizontal motility and chin-up position of the head.

A

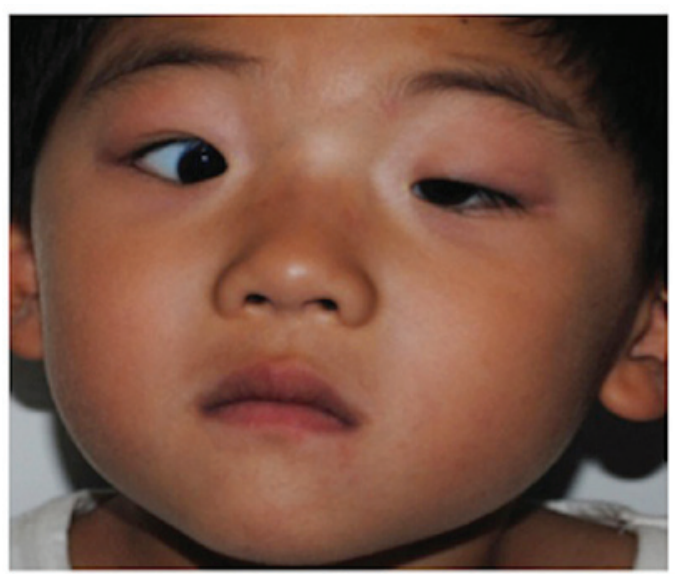

C

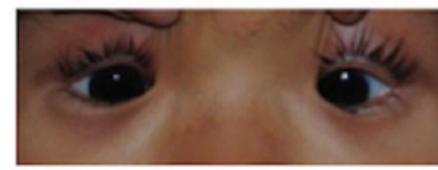

F

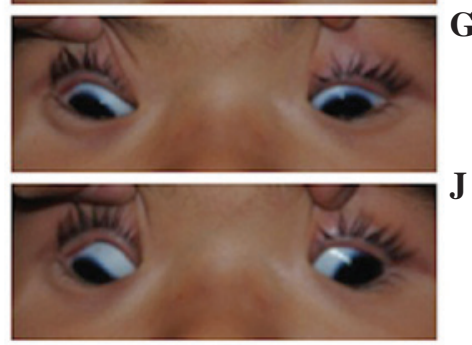

D

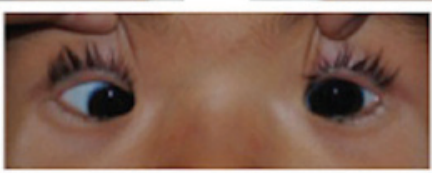

G

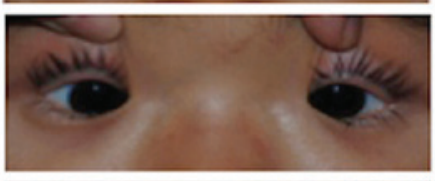

B

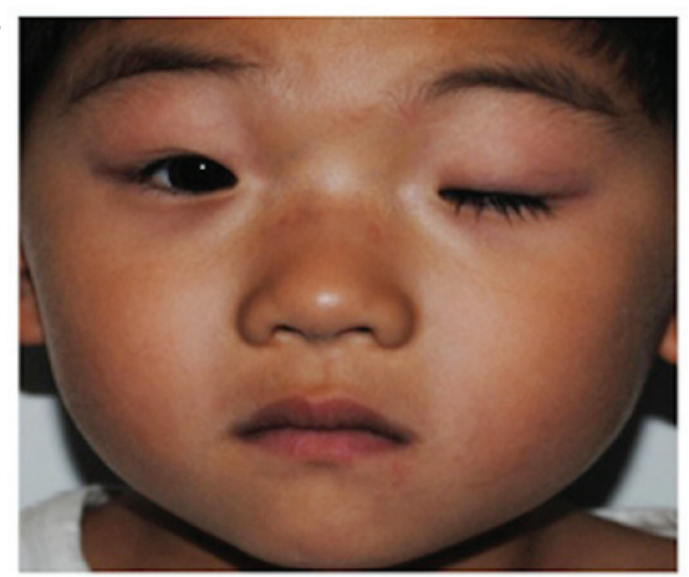

E

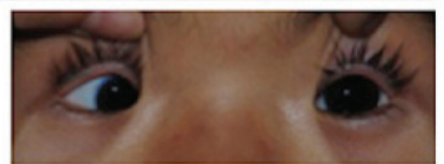

H

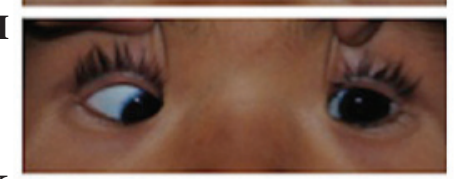

K

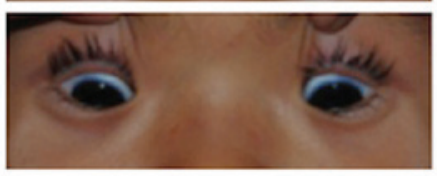

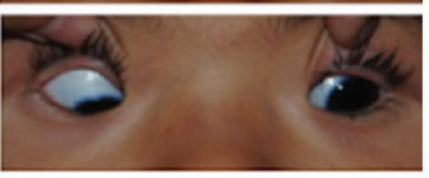

Figure 3. Images of the proband from family 2. Images were captured of the (A) compensatory head position, in (B) primary gaze with unaltered eyelids and in (C-K) nine-gaze positions with lifted eyelids (in sequence C-K, looking up and right, straight up, up and left, straight right, straight, straight left, down and left, straight down, down and left). This individual exhibited typical CFEOM3 features, including unilateral ptosis, variable restricted upgaze and horizontal eye movements, with an ability to elevate the left eye above the midline, and chin-up position of the head. 


Family 2: II:1

Figure 4. Range of extraocular movement and position of the eyes in the primary position of the patients in the two families. Extraocular movement was rated on a scale of $0-4$, with 0 indicating no movement in that direction and 4 indicating normal movement in that direction. The grey dot indicates the position of the pupil within the orbit in primary gaze. SR, superior rectus; IR, inferior rectus; LR, lateral rectus; MR, medial rectus; SO, superior oblique; IO, inferior oblique; OD, oculus dexter (the right eye); OS, oculus sinister (the left eye).
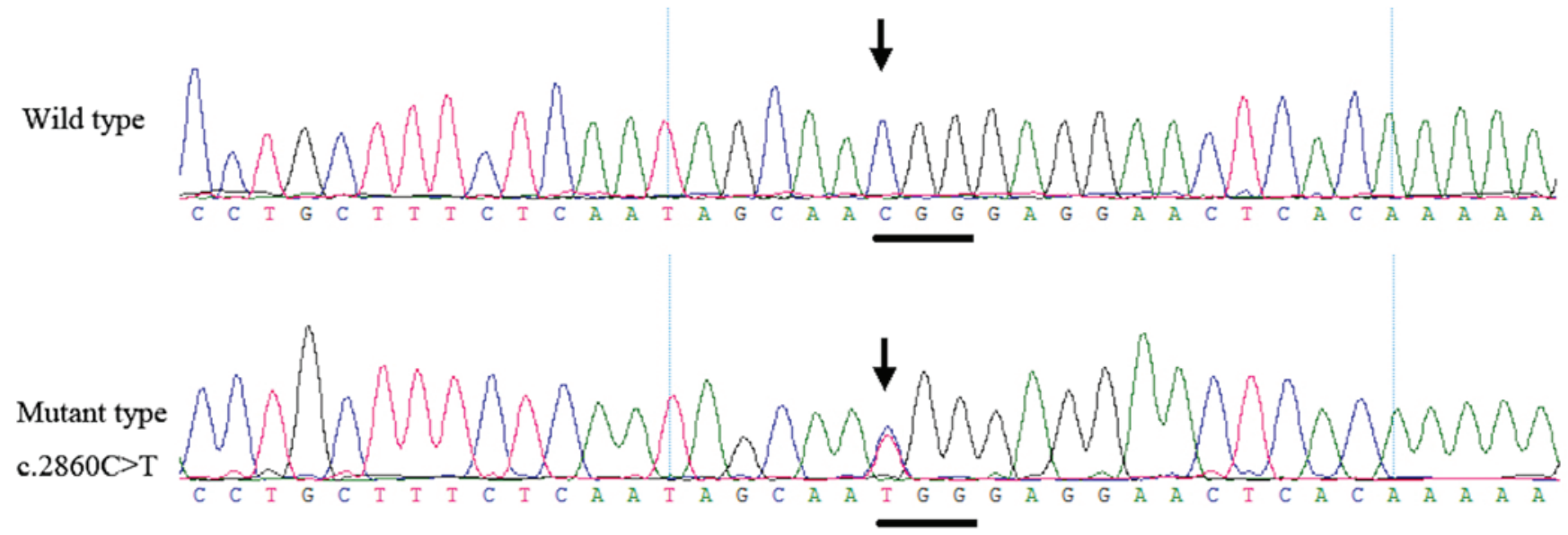

Figure 5. Sequencing chromatograms showing KIF21A mutations in the four affected members of family 1 and family $2(2860 \mathrm{C}>\mathrm{T})$. The position of the mutation is marked by an arrow and the corresponding wild-type sequence is shown as a control. 
In the present study, a heterozygous $K I F 21 A$ c. $2860 C>T$ mutation was found to be present in a family with CFEOM1 and a family with CFEOM3. Identical gene mutations causing distinct phenotypes were observed in CFEOM. Taken together, it is reasonable to conclude that the KIF21A mutation may be a major disease-causing gene for CFEOM3 in Chinese individuals.

\section{References}

1. Brown HW: Congenital structural muscle anomalies. In: Strabismus Ophthalmic Symposium. Allen JH (ed). CV Mosby, St Louis, pp205-236, 1950.

2. Nakano M, Yamada K, Fain J, Sener EC, Selleck CJ, Awad AH, Zwaan J, Mullaney PB, Bosley TM and Engle EC: Homozygous mutations in ARIX (PHOX2A) result in congenital fibrosis of the extraocular muscles type 2. Nat Genet 29: 315-320, 2001.

3. Tischfield MA, Baris HN, Wu C, Rudolph G, Van Maldergem L, He W, Chan WM, Andrews C, Demer JL, Robertson RL, et al: Human TUBB3 mutations perturb microtubule dynamics, kinesin interactions and axon guidance. Cell 140: 74-87, 2010.

4. Yamada K, Andrews C, Chan WM, McKeown CA, Magli A, de Berardinis T, Loewenstein A, Lazar M, O'Keefe M, Letson R, et al: Heterozygous mutations of the kinesin KIF21A in congenital fibrosis of the extraocular muscles type 1 (CFEOM1). Nat Genet 35: 318-321, 2003.

5. Lu S, Zhao C, Zhao K, Li N and Larsson C: Novel and recurrent KIF21A mutations in congenital fibrosis of the extraocular muscles type 1 and 3. Arch Ophthalmol 126: 388-394, 2008.

6. Traboulsi EI and Engle EC: Mutations in KIF21A are responsible for CFEOM1 worldwide. Ophthalmic Genet 25: 237-239, 2004.

7. Chan WM, Andrews C, Dragan L, Fredrick D, Armstrong L, Lyons C, Geraghty MT, Hunter DG, Yazdani A, Traboulsi EI, et al: Three novel mutations in KIF21A highlight the importance of the third coiled-coil stalk domain in the etiology of CFEOM1. BMC Gent 8: 26, 2007.

8. Engle EC: The molecular basis of the congenital fibrosis syndromes. Strabismus 10: 125-128, 2002.

9. Yamada K, Chan WM, Andrews C, Bosley TM, Sener EC, Zwaan JT, Mullaney PB, Ozturk BT, Akarsu AN, Sabol LJ, et al: Identification of KIF21A mutations as a rare cause of congenital fibrosis of the extraocular muscles type 3 (CFEOM3). Invest Ophthalmol Vis Sci 45: 2218-2223, 2004.

10. Doherty EJ, Macy ME, Wang SM, Dykeman CP, Melanson MT and Engle EC: CFEOM3: A new extraocular congenital fibrosis syndrome that maps to $16 \mathrm{q} 24.2-\mathrm{q} 24.3$. Invest Ophthalmol Vis Sci 40: 1687-1694, 1999.

11. Gillies WE, Harris AJ, Brooks AM, Rivers MR and Wolfe RJ: Congenital fibrosis of the vertically acting extraocular muscles. A new group of dominantly inherited ocular fibrosis with radiologic findings. Ophthalmology 102: 607-612, 1995.

12. Mackey DA, Chan WM, Chan C, Gillies WE, Brooks AM, O'Day J and Engle EC: Congenital fibrosis of the vertically acting extraocular muscles maps to the FEOM3 locus. Human Genet 110: 510-512, 2002.

13. Sener EC, Lee BA, Turgut B, Akarsu AN and Engle EC: A clinically variant fibrosis syndrome in a Turkish family maps to the CFEOM1 locus on chromosome 12. Arch Ophthalmol 118: 1090-1097, 2000.
14. Khan AO, Shinwari J, Omar A, Al-Sharif L, Khalil DS, Alanazi M, Al-Amri A and Al Tassan N: Lack of KIF21A mutations in congenital fibrosis of the extraocular muscles type I patients from consanguineous Saudi Arabian families. Mol Vis 17: 218-224, 2011.

15. Engle EC, Kunkel LM, Specht LA and Beggs AH: Mapping a gene for congenital fibrosis of the extraocular muscles to the centromeric region of chromosome 12. Nat Genet 7: 69-773, 1994.

16. Engle EC, Marondel I, Houtman WA, de Vries B, Loewenstein A, Lazar M, Ward DC, Kucherlapati R and Beggs AH: Congenital fibrosis of the extraocular muscles (autosomal dominant congenital external ophthalmoplegia): Genetic homogeneity, linkage refinement, and physical mapping on chromosome 12. Am J Hum Genet 57: 1086-1094, 1995.

17. Engle EC, McIntosh N, Yamada K, Lee BA, Johnson R, O'Keefe M, Letson R, Lodon A, Ballard E, Ruttum M, et al: CFEOM1, the classic familial form of congenital fibrosis of the extraocular muscles, is genetically heterogeneous but does not result from mutations in ARIX. BMC Genet 3: 3, 2002.

18. Uyama E, Yamada K, Kawano H, Chan WM, Andrews C, Yoshioka M, Uchino M and Engle EC: A Japanese family with FEOM1-linked congenital fibrosis of the extraocular muscles type 1 associated with spinal canal stenosis and refinement of the FEOM1 critical region. Neuromuscul Disord 13: 472-478, 2003.

19. Miki H, Setou M, Kaneshiro K and Hirokawa N: All kinesin superfamily protein, KIF, genes in mouse and human. Proc Natl Acad Sci USA 98: 7004-7011, 2001.

20. Yamada K, Hunter DG, Andrews C and Engle EC: A novel KIF21A mutation in a patient with congenital fibrosis of the extraocular muscles and Marcus Gunn jaw-winking phenomenon. Arch Ophthalmol 123: 1254-1259, 2005.

21. Wang P, Li S, Xiao X, Guo X and Zhang Q: KIF21A novel deletion and recurrent mutation in patients with congenital fibrosis of the extraocular muscles-1. Int J Mol Med 28: 973-975, 2011

22. Li ND, Zhao J, Wang LM, Chen X, Ma HZ, Zhu LN, Guo X and Zhao KX: R954 mutations in KIF21A gene in Chinese patients with congenital fibrosis of extraocular muscles. Zhonghua Yan Ke Za Zhi 48: 1077-1082, 2012 (In Chinese).

23. Ali M, Venkatesh C, Ragunath A and Kumar A: Mutation analysis of the KIF21A gene in an Indian family with CFEOM1: Implication of $\mathrm{CpG}$ methylation for most frequent mutations. Ophthalmic Genet 25: 247-255, 2004.

24. Pfeifer GP: Mutagenesis at methylated CpG sequences. Curr Top Microbiol Immunol 301: 259-281, 2006.

25. Lin LK, Chien YH, Wu JY, Wang AH, Chiang SC and Hwu WL: KIF21A gene c. $2860 \mathrm{C}>\mathrm{T}$ mutation in congenital fibrosis of extraocular muscles type 1 and 3 . Mol Vis 11: 245-248, 2005.

26. Yang X, Yamada K, Katz B, Guan H, Wang L, Andrews C, Zhao G, Engle EC, Chen H, Tong Z, et al: KIF21A mutations in two Chinese families with congenital fibrosis of the extraocular muscles (CFEOM). Mol Vis 16: 2062-2070, 2010.

27. Zhang J, Zhou L, Zha Y, Liu T, Tian M, Yuan J and Xing Y: The gene mutation screening of a family with congenital fibrosis of the extraocular muscles associated with corpus callosum agenesis. Zhonghua Yan Ke Za Zhi 49: 621-626, 2013 (In Chinese). 